\title{
Tamsulosin Can Improve Lower Urinary Tract Symptoms in Patients Under Active Surveillance Due to Low Risk Prostate Cancer: Prospective Controlled Study
} \author{
(1) Kaya Horasanlı MD ${ }^{1}$ \\ ' Şişli Hamidiye Etfal Training and Research Hospital, Clinic of Urology, Istanbul, Turkey \\ 2 Sancaktepe Şehit Prof. Dr. IIhan Varank Training and Research Hospital, Clinic of Urology, Istanbul, Turkey \\ ${ }^{3}$ Ankara Training and Research Hospital, Clinic of Urology, Ankara, Turkey \\ ${ }^{4}$ Mersin Medical Park Hospital, Clinic of Urology, Mersin, Turkey \\ 5 Istinye University Faculty of Medicine, Ulus Liv Hospital, Clinic of Urology, Istanbul, Turkey
}

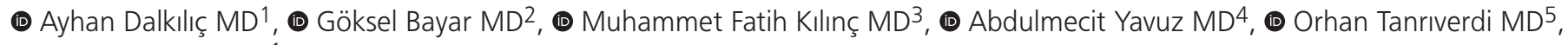

\begin{abstract} (AS) for low-risk prostate cancer (Pca). follow-up. and the need for surgical treatment were similar to the control group.

Keywords: Prostate cancer, active surveillance, lower urinary tract symptoms, tamsulosin

\section{Introduction}

Prostate cancer (Pca) is the most common non-skin cancer among men (1). Currently, prostate-specific antigen (PSA) and multiparametric magnetic resonance imaging (mpMRI) are commonly used, and approximately $90 \%$ potential cure can be ensured in patients newly diagnosed with localized Pca (2). Treatments that may be applied in this stage are radical prostatectomy (RP) and radiotherapy (RT) (3). Apart from these,
\end{abstract}

Objective: We aimed to evaluate the efficacy of tamsulosin in the treatment of lower urinary tract symptoms (LUTS) in patients under active surveillance

Materials and Methods: Patients who underwent prostate biopsy between 2010 and 2014 were evaluated prospectively. Inclusion criteria for AS were PSA level below $10 \mathrm{ng} / \mathrm{mL}$, a tumor in a maximum of two cores, a tumor core percentage below 50\%, a clinical stage $\leq$ T2a and a Gleason score $\leq 6$. Patients under AS with LUTS were included in the study group and patients with benign pathology results with LUTS were included in the control group. International prostate symptom score (IPSS) and uroflowmetry test were used to evaluate LUTS. The maximum flow rate ( $Q_{\text {max }}$ ) was recorded. All patients received tamsulosin $0.4 \mathrm{mg}$ once daily in a modified-release formulation (Flomax MRß, Astellas, Japan). Transurethral resection prostatectomy (TUR-P) was performed when surgical treatment was necessary for obstruction despite tamsulosin treatment.

Results: The study included a total of 91 patients, 41 patients in the AS group and 50 patients in the control group. Patients were assessed after six months. There was a $30 \%$ reduction in IPSS in the AS group and $24.5 \%$ in the control group $(p=0.591) . Q_{\max }$ increased by $20.4 \%$ in the AS group and by $20 \%$ in the control group ( $p=0.985)$. The need for TUR-P was similar between the two groups $(14.6 \%$ vs $20 \%, p=0.503)$ during three-year

Conclusion: Tamsulosin can be used safely and with high efficacy for LUTS in patients under AS for low-risk Pca. The improvement in IPSS and $\mathrm{Q}_{\max }$

low-risk patients should not undergo curative treatment and there is the option of active surveillance (AS), which is defined as delay until certain progression criteria apply (3). AS is based on periodically repeated PSA tests, digital rectal examination (DRE) and control biopsy, and the main aim is to postpone or even avoid definitive treatment without disease progression (4). Lower urinary tract symptoms (LUTS) associated with benign prostate enlargement observed in adult males involve complaints that disrupt the quality of life (QoL). These complaints are storage

This study was presented at $4^{\text {th }}$ Urological Surgery Society Congress in 2018 as an oral presentation.

Address for Correspondence: Göksel Bayar MD, Sancaktepe Şehit Prof. Dr. İlhan Varank Training and Research Hospital, Clinic of Urology, İstanbul, Turkey Phone: +90 2166063300 E-mail: goxelle@yahoo.com ORCID-ID: orcid.org/0000-0003-1506-9732 Received: 25.12.2018 Accepted: 25.12.2018 
(frequent urination, night-time urination and sudden urgency), voiding (difficulty beginning urination, pain and discomfort during urination) and post-voiding symptoms (dripping after urination) (5). LUTS are defined by two different symptom frequencies of at least "sometimes" and at least "often" for all LUTS, except incontinence with at least "a few times" per month and at least "a few times" per week. Therefore, the overall prevalence of LUTS is approximately $72 \%$ and moderatesevere LUTS is $48 \%$ in men older than 40 years (6).

Although palliative (medication, lifestyle advice etc.) treatment improves LUTS, curative treatments (RP, RT and brachytherapy) worsen LUTS in patients with localized Pca (7). Tamsulosin is an effective and reliable drug used for all age groups in men with LUTS (8). It has been shown to reduce international prostate symptom score (IPSS) and residual urine, and increase maximum urinary flow rate $\left(\mathrm{Q}_{\max }\right)$ in advanced stage $\mathrm{Pca}$ patients with LUTS (9).

We aimed to evaluate the efficacy and safety of tamsulosin treatment for LUTS in patients under AS patients for low-risk Pca.

\section{Materials and Methods}

Patients who underwent prostate biopsy at our clinic between June 2010 and November 2014 were investigated prospectively. Prostate biopsy was performed due to elevated PSA (>2.5 g/ $\mathrm{mL}$ ) and/or abnormality in DRE. Those with prostate biopsy results indicating Pca appropriate for AS were offered the AS option. Inclusion criteria for AS were PSA level below $10 \mathrm{ng} /$ $\mathrm{mL}$, tumor in a maximum of two cores, tumor core percentage below $50 \%$, clinical stage $\leq \mathrm{T} 2 \mathrm{a}$ and Gleason score (GS) $\leq 6$. AS was not offered to patients below the age of 50 and above the age of 75. Patients appropriate for AS regarding biopsy results, DRE findings and PSA level criteria were informed about AS along with definitive treatments. Re-biopsy was performed within a maximum of 3 months in patients who accepted AS. Patients with re-biopsy results appropriate for AS were included. Among these patients, people with LUTS were included in the study. PSA levels of the patients were evaluated and DRE was performed at six-month intervals. Control biopsy was performed one year later and every three years thereafter. All biopsies were performed under the guidance of transrectal ultrasound (TRUS). The first biopsy had 12 cores, while re-biopsies and annual check-up biopsies had 20 cores. The definitive treatment criteria were determined as GS upgrade on prostate biopsy, tumor core percentages above $50 \%$ or more than two tumor cores. Patients requiring definitive treatment (RP or RT) during AS were excluded from the final analysis (Figure 1).

Patients with benign biopsy results but without LUTS, patients with a surgical decision before biopsy (recurrent retention, macroscopic hematuria etc.), patients on LUTS drug before biopsy, patients on drugs other than alpha blockers (such as tadalafil for erectile dysfunction), and patients who did not participate (like not accepting long-term follow-up or living in other cities) or patients who were inappropriate due to paramedical reasons (insufficient intellectual level, living in rural areas, etc.) were not included in the study. All remaining patients were included in the study as the control group (Figure 1).
Prostate volumes were measured transrectally during biopsy and no additional imaging (such as mpMRI) was performed. Patients in both groups initially completed the IPSS form, QoL score, and underwent uroflowmetry test to record Qmax. Patients with no symptoms other than nocturia in the IPSS form were not included in the study, as this was not accepted as prostateassociated LUTS. Patients without LUTS or with mild LUTS (1-7 points in IPSS) were not included in the study. All patients received tamsulosin $0.4 \mathrm{mg}$ once daily in a modified-release formulation (Flomax MR®, Boehringer Ingelheim, Germany). None of the patients received 5-alpha reductase inhibitors, anticholinergic drugs or phosphodiesterase- 5 inhibitors, as these drugs may improve LUTS and cause confusion to the results of the study. Patients were called for LUTS examination at the $6^{\text {th }}$ and $12^{\text {th }}$ month of the first year and annually after one year. During each control, vital signs (blood pressure, pulse) were measured, side effects related to any drug were questioned, IPSS form was filled and uroflowmetry test with Qmax was recorded. During the controls, patients continued to use the drug and had used the drug on the day of the control. On check-up, TUR-P was performed if the following parameters were observed despite medical treatment: IPSS score above 20 (mainly obstructive symptoms), $Q_{\max }$ value not increasing above $10 \mathrm{~mL} / \mathrm{s}$, recurrent macroscopic hematuria, recurrent urinary tract infection, development of dilatation in the upper urinary tract, recurrent urinary retention, overflow incontinence, bladder diverticulum or stone formation. Due to possibility of subsequent definitive treatment requirement, open prostatectomy was avoided as it makes RP technically difficult. No TUR-P procedure was performed without the patient being included in the study for at least one year. IPSS and uroflowmetry values were not included in the final analysis after TUR-P operations (Ref no: 0671-5636).

\section{Statistical Analysis}

The minimum number of patients required was calculated by assuming that a mean difference of $10 \%$ between the two groups was significant with $80 \%$ accuracy and $5 \%$ error. The minimum number of patients in each group was calculated as 40. Due to the possibility of need for definitive treatment or loss during follow-up, the patient number in each group was determined as 55. Age, PSA values, prostate volumes, IPSS score, Qmax value and surgical treatment requirements were compared between the two groups. Student's t-test was used to compare the numerical data between the two groups, and Wilcoxon analysis was used to compare the variation in data over time within the groups. Pearson chi-square test was used for non-numerical data. A $p$ value less than 0.05 was accepted as significant.

\section{Results}

A total of 410 prostate biopsy results were assessed, 170 (41.4\%) were malignant and 240 (58.6\%) were benign. After exclusion of other patients, AS group included a total of 55 patients. However, during follow-up, nine patients received definitive treatment (seven patients due to pathological upgrade and two due to patient anxiety) and five patients died, so 41 patients were included in the final analysis. The control group 


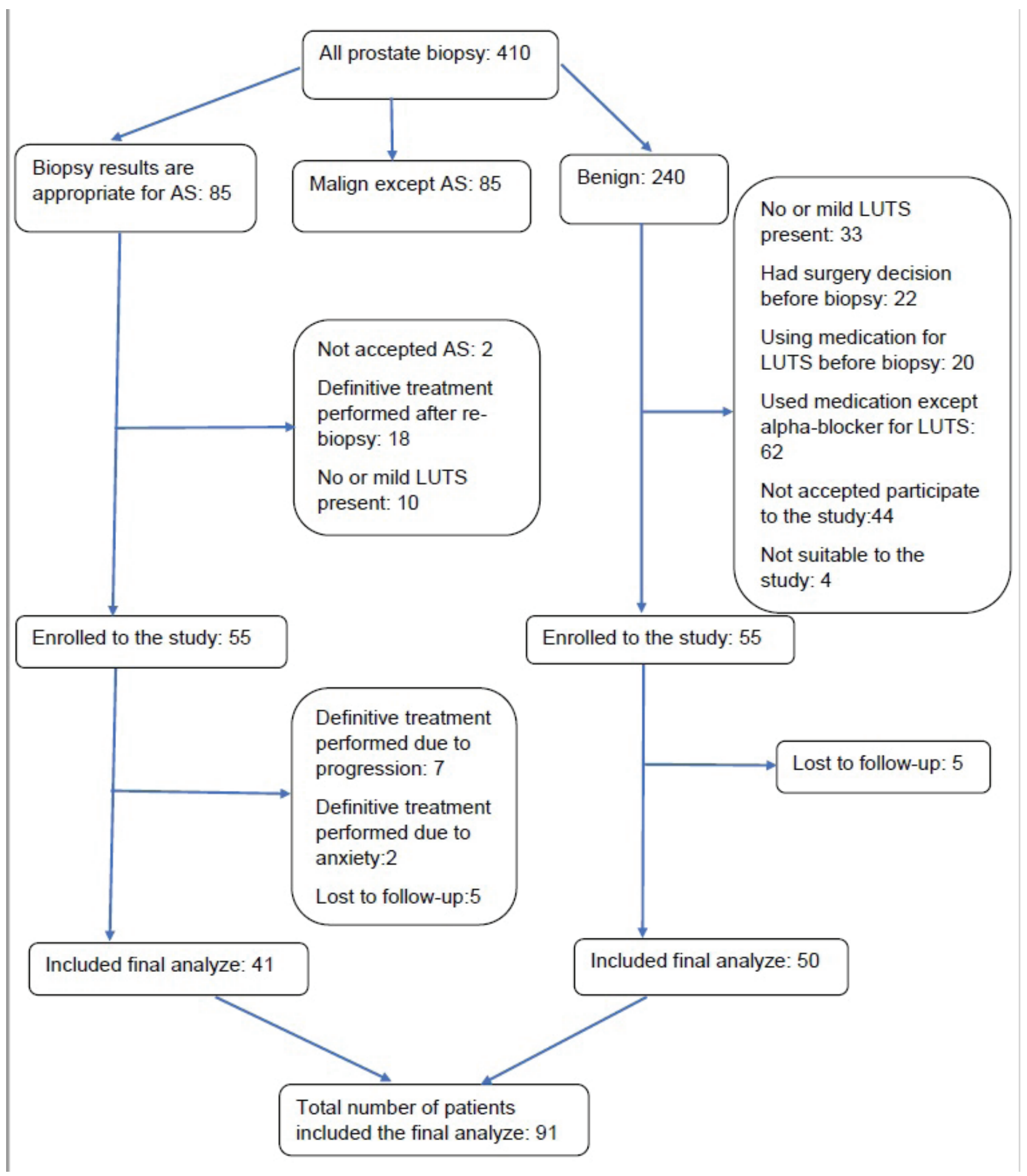

Figure 1. Figure shows inclusion and exclusion criteria and numbers (AS: Active surveillance, LUTS: Lower urinary tract symptoms)

included 55 patients. During follow-up, five patients died and the analysis included 50 patients. The mean follow-up was 5.3 (range $=3-7$ ) years. In the control group, the number of patients who had increased PSA level and who underwent second biopsy was eight. No Pca was identified in any of these patients. The study results included a total of 91 patients; 41 in the AS group and 50 in the control group (Figure 1). The mean age of the patients was 61.4 years $($ range $=50-72)$. The mean PSA value was 4.74 (range $=2.7-9$ ). Ten patients reported side effects related to the drug. These included dizziness and ejaculatory dysfunction. Patients were informed about side effects and told what to do; therefore, no patient discontinued the drug due to 
side effects. A total of 16 patients required TUR-P due to LUTS. All TUR-P pathologies were reported as benign.

When the two groups were compared, the mean age and prostate volumes were similar. Although the mean PSA value was significantly higher in the AS group, it was moderate. In the first application, mean IPSS and $\mathrm{Q}_{\max }$ values were similar in both groups. After 6 months of tamsulosin treatment, the change in IPSS, QoL score and $\mathrm{Q}_{\max }$ scores were similar in both groups. Surgical requirements developed in six patients in the AS group and 10 patients in the control group, and the rates were statistically similar. Drug-related side effects were reported in four patients in the AS group and six patients in the control group, and the rates were similar (Table 1).

The change in mean IPSS score is shown in Figure 1. There was a $30 \%$ reduction in IPSS scores in the AS group and $24.5 \%$ reduction in the control group compared to first administration after tamsulosin use. Then, the mean IPSS score increased slightly in both groups during the year and formed a plateau (Figure 2).

The mean $\mathrm{Q}_{\max }$ change is shown in Figure 2. After tamsulosin administration, there was a $20.4 \%$ increase in the AS group and $20 \%$ increase in the control group compared to the first administration. Later during the year, there was a slightly decreasing plateau in parallel in both groups (Figure 3).

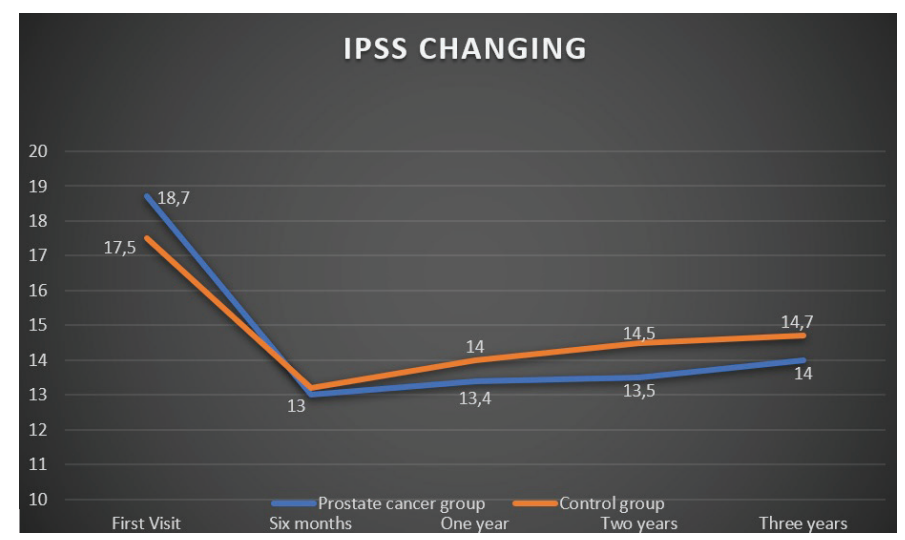

Figure 2. IPSS change of two groups over time (IPSS: International Prostate Symptom Score)

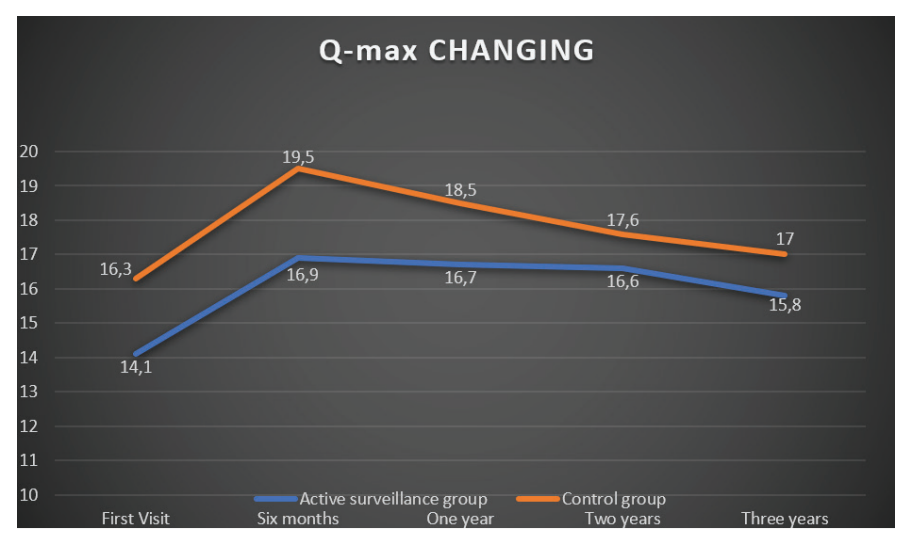

Figure 3: Maximum urinary flow rate $\left(Q_{\max }\right)$ change of two groups over time

\section{Discussion}

AS for low-risk Pca was first proposed by Klotz et al. (10). When long-term results from different clinics and high numbers of patients have been published, there is high cancer-specific survival (above $90 \%$ ) and comorbidities caused by treatment are avoided as patients are not administered any medication $(11,12,13)$. A study in Canada showed that more than three quarters of patients abiding by AS criteria chose AS (14). When RP is compared with AS for patients with local stage Pca, RP was found to be beneficial in patients with PSA above $10 \mathrm{ng} / \mathrm{mL}$ or with high-risk Pca, however, the 10-year survival rates for low-risk patients appear to be similar (15). During AS, the progression-related definitive treatment requirements are $10-35 \%(16,17)$. In our study, seven patients $(14 \%)$ had definitive treatment due to progression (three patients had RP, four patients had RT). Apart from progression, patients may insist on definitive treatment due to anxiety leading to definitive treatment for $10 \%$ of patients (18). In our study, two patients (4\%) had RP performed due to patient insistence in spite of no progression.

LUTS are a cluster of symptoms that is commonly seen, uncomfortable and disrupts QoL (19). LUTS has a wide range of treatment from lifestyle changes to open surgery and whatever the applied treatment, QoL increases due to improvements in symptoms (20). LUTS displays a dynamic situation over time; it worsens in some patients, remains the same in others and improves in some (21). However, the rate of patients with improvement in symptoms without treatment is very low (22). As a result, though surgical treatment is not performed in every patient, at least, lifestyle changes and/or drug treatment is recommended.

Tamsulosin binds to alpha- 1 receptors showing antagonistic

\begin{tabular}{|c|c|c|c|}
\hline & $\begin{array}{l}\text { Active } \\
\text { Surveillance } \\
\text { Group }\end{array}$ & $\begin{array}{l}\text { Control } \\
\text { Group }\end{array}$ & p \\
\hline Number of patients & 41 & 50 & \\
\hline Mean age (year) $\pm S D$ & $61.5 \pm 5.4$ & $61.3 \pm 6.7$ & 0.981 \\
\hline Mean PSA $(\mathrm{ng} / \mathrm{mL}) \pm \mathrm{SD}$ & $5.2 \pm 1.8$ & $4.3 \pm 1.3$ & $0.009^{*}$ \\
\hline Mean prostate volume $(\mathrm{cc}) \pm \mathrm{SD}$ & $41 \pm 12$ & $47 \pm 17$ & 0.41 \\
\hline Mean IPSS at first visit \pm SD & $18.7 \pm 6.9$ & $18.5 \pm 7.4$ & 0.806 \\
\hline $\begin{array}{l}\text { Mean IPSS change after six } \\
\text { months (percent of first IPSS) }\end{array}$ & $30.5 \pm 17.7$ & $24.5 \pm 19.6$ & 0.591 \\
\hline Mean QoL score change \pm SD & $1.75 \pm 0.4$ & $1.63 \pm 0.35$ & 0.42 \\
\hline $\begin{array}{l}\text { Mean } Q \max (\mathrm{mL} / \mathrm{sn}) \\
\text { at first visit } \pm \mathrm{SD}\end{array}$ & $14 \pm 4.3$ & $15.1 \pm 5$ & 0.152 \\
\hline $\begin{array}{l}\text { Mean Qmax change after six } \\
\text { months (percent of first } Q_{\max } \text { ) }\end{array}$ & $20.4 \pm 26.9$ & $20 \pm 22.5$ & 0.985 \\
\hline Need for surgery (\%) & $14.6 \%$ & $20 \%$ & 0.503 \\
\hline Adverse reaction (\%) & $4(9.75)$ & $6(12)$ & 0.733 \\
\hline \multicolumn{4}{|c|}{$\begin{array}{l}\text { SD: Standard deviation, PSA: Prostate specific antigen, IPSS: International } \\
\text { Prostate Symptom Score, QoL: Quality of life, Qmax: Maximum urination flow } \\
\text { rates } \\
{ }^{*} \text { indicates a statistically significant } p \text { value }<0.05 \text {. }\end{array}$} \\
\hline
\end{tabular}


properties and causes relaxation of smooth muscles; thus, LUTS improve through expansion of the prostatic urethra and bladder neck (23). Regarding Combat study, there was a $30 \%$ reduction in IPSS score after three months in the tamsulosinonly group; however, the IPSS score increased slightly in the following months, and at the end of four years, the change in IPSS decreased to $23 \%$ (23). In our study, IPSS score reduced by $30 \%$ in AS patients after six months of tamsulosin treatment; however, IPSS increased slightly in the following months, and at the end of three years, there was a reduction of $25 \%$ compared to the initial IPSS score. In the Combat study, patients receiving tamsulosin alone had a $10 \%$ increase in $\mathrm{Q}_{\max }$ values in the first month with a slight decrease over the months and a $7 \%$ increase by the end of four years (23). In our study, mean $\mathrm{Q}_{\max }$ value in AS patients increased by $20.4 \%$ at the end of the six months; however, there was a decline in the following months, and at the end of three years, there was a $12 \%$ increase compared to first application. After six months, there is no further improvement in IPSS and $Q_{\max }$ so this can give us a hint to decide the time of surgery. The IPSS reduction and $\mathrm{Q}_{\max }$ increase rates obtained in our study were parallel to the change over the years in the Combat study; however, our study obtained better improvements. However, it should not be forgotten that the efficacy of tamsulosin decreases as the patient age increases (8). In our study the mean age of patients was 61.4 years, while it was 66.2 years in the Combat study (23). As a result, the better improvement in IPSS and $\mathrm{Q}_{\max }$ values in our study compared to the Combat study may possibly be due to the lower mean patient age in our study. The addition of tamsulosin to androgen deprivation therapy (ADT) in patients with advanced stage Pca improved LUTS $(9,24)$. The addition of tamsulosin to ADT in advanced stage Pca patients ensured a $50 \%$ improvement in IPSS and a $40 \%$ increase in $\mathrm{Q}_{\max }$ (25). However, tamsulosin alone is not responsible for this objective amelioration in LUTS, because patients not receiving tamsulosin but just ADT obtained the same results but over a longer period (25). ADT is probably responsible for excessive improvement in IPSS and $\mathrm{Q}_{\max }$ values.

Patients with LUTS require surgery due to benign prostate hyperplasia-related obstruction at a rate of $10-24 \%$ over 3 years $(24,26)$. Surgery was required in $14.6 \%$ of AS patients and $20 \%$ of the control group. The rate of surgical requirement in our study was in parallel with the literature. RP results performed after TUR-P are similar to the results of patients in whom TUR-P is not performed (27). PSA elevation that causes anxiety in AS patients and unnecessary definitive treatment demands can be controlled by TUR-P; therefore, anxiety caused by urinary symptoms and PSA elevation improves (28). The benefits of AS and control group patients with performed TUR-P were similar.

The long-term use of 5-alpha reductase inhibitors reduces surgical requirements due to benign prostate hyperplasia by $50 \%$ (26). Administration of dutasteride to AS patients does not cause progression (29). In fact, it may even delay progression slightly (30). However, dutasteride increases the apparent diffusion coefficient of the tumor region in AS patients in mpMRI and causes uncertainty about the tumor area (31). As a result, AS patients should be careful about the use of dutasteride before $\mathrm{mpMRI}$ is performed or if $\mathrm{mpMRI}$ is going to be used during follow-up. All these studies were published after we initiated our study. As a result, there was insufficient data at the beginning of the study and the use of 5-alpha reductase inhibitors was avoided to prevent difficulties in PSA follow-up.

In our study, the number of patients seems to be a limitation and there may be a need for more patients for higher accuracy and stronger power values. Residual urine amounts after urination can only be accurately measured by using a catheter, and we believe that inserting a catheter after each uroflowmetry is excessively invasive. As a result, though residual urine after urination measurements are used in our clinic, we did not include this data in the study. We did not find any studies on the use of tamsulosin or another alpha-blocker for the treatment of LUTS in AS patients in PubMed and Cochrane databases as of December 2017. As a result, no direct comparisons were made. Therefore, we used studies that included advanced Pca patients. Detecting tumor volume may be important in AS patients via mpMRI, but it was not in routine use eight years ago; this might be another limitation of the study.

\section{Conclusion}

Tamsulosin can be used safely and with high efficacy in the treatment of LUTS in low-risk Pca patients under AS. The improvement in IPSS and $\mathrm{Q}_{\max }$ obtained by tamsulosin treatment and the need for surgical treatment were similar between AS and control patients.

\section{Ethics}

Ethics Committee Approval: Ankara Training and Research Hospital Local Ethic Committee (Ref no: 0671-5636).

Informed Consent: Informed consent was obtained from all participants.

Peer-review: Externally peer-reviewed.

\section{Authorship Contributions}

Surgical and Medical Practices: O.T., A.D., K.H. Concept: G.B., O.T. Design: G.B., M.F.K., A.Y., Data Collection or Processing: M.F.K., A.M.Y. Analysis or Interpretation: G.B., O.T., K.H., A.D., Literature Search: A.Y., M.F.K Writing: G.B., A.D.

Conflict of Interest: No conflict of interest was declared by the authors.

Financial Disclosure: The authors declared that this study received no financial support.

\section{References}

1. Siegel RL, Miller KD, Jemal A. Cancer statistics, 2016. CA Cancer J Clin 2016;66:7-30.

2. Miller KD, Siegel RL, Lin CC, et al. Cancer treatment and survivorship statistics, 2016. CA Cancer J Clin 2016;66:271-289.

3. Mottet N, Bellmunt J, Briers E, et al. EAU-ESTRO-ESUR-SIOC Guidelines on Prostate Cancer 2017. Available at: http://uroweb.org/ guideline/prostate-cancer.

4. Klotz L. Active surveillance for prostate cancer: for whom? J Clin Oncol 2005;23:8165-8169.

5. Gravas S, Bach T, Drake M, et al. EAU Guidelines on Management of Non-Neurogenic Male Lower Urinary Tract Symptoms (LUTS), 
incl. Benign Prostatic Obstruction (BPO) 2017. Available at: http:// uroweb.org/guideline/treatment-of-non-neurogenic-male-luts.

6. Coyne KS, Sexton CC, Thompson CL, et al. The prevalence of lower urinary tract symptoms (LUTS) in the USA, the UK and Sweden: results from the Epidemiology of LUTS (EpiLUTS) study. BJU Int 2009;104:352-360.

7. van Andel G, Bottomley A, Fosså SD, et al. An international field study of the EORTC QLQ-PR25: a questionnaire for assessing the healthrelated quality of life of patients with prostate cancer. Eur J Cancer 2008;44:2418-2424.

8. Michel MC, Mehlburger L, Bressel HU, Goepel M. Comparison of tamsulosin efficacy in subgroups of patients with lower urinary tract symptoms. Prostate Cancer Prostatic Dis 1998;1:332-335.

9. Govorov AV, Vasilyev AO, Pushkar DY. Efficacy of tamsulosin for treating lower urinary tract symptoms in patients with advanced prostate cancer. Urologiia 2017;4:37-41.

10. Klotz LH, Choo R, Morton G, Danjoux C. Expectant management with selective delayed intervention for favorable-risk prostate cancer. Can J Urol 2002;9(Suppl 1):2-7.

11. Klotz, Vesprini $D$, Sethukavalan $P$, et al. Long-term follow-up of a large active surveillance cohort of patients with prostate cancer. J Clin Oncol 2015;33:272-277.

12. Adamy A, Yee DS, Matsushita K, et al. Role of prostate specific antigen and immediate confirmatory biopsy in predicting progression during active surveillance for low risk prostate cancer. J Urol 2011;185:477-482.

13. Khatami A, Aus G, Damber JE, et al. PSA doubling time predicts the outcome after active surveillance in screening-detected prostate cancer: results from the European randomized study of screening for prostate cancer, Sweden section. Int J Cancer 2007;120:170-174.

14. Timilshina $N$, Ouellet $V$, Alibhai SM, et al. Analysis of active surveillance uptake for low-risk localized prostate cancer in Canada: a Canadian multi-institutional study. World J Urol 2017;35:595-603.

15. Bill-Axelson A, Holmberg L, Ruutu M, et al. Radical prostatectomy versus watchful waiting in early prostate cancer. N Engl J Med 2011;5:364:1708-1717.

16. Dall'Era MA, Konety BR, Cowan JE, et al. Active surveillance for the management of prostate cancer in a contemporary cohort. Cancer 2008; 15;112:2664-2670.

17. Soloway MS, Soloway CT, Eldefrawy A, et al. Careful selection and close monitoring of low-risk prostate cancer patients on active surveillance minimizes the need for treatment. Eur Urol 2010;58:831-835.

18. Klotz L, Zhang L, Lam A, et al. Clinical results of long-term follow-up of a large, active surveillance cohort with localized prostate cancer. J Clin Oncol 2010;28:126-131.

19. Agarwal A, Eryuzlu LN, Cartwright R, et al. What is the most bothersome lower urinary tract symptom? Individual- and population-level perspectives for both men and women. Eur Urol 2014;65:1211-1217.
20. De Ridder $D$, Roumeguère $T$, Kaufman L. Urgency and other lower urinary tract symptoms in men aged $\geq 40$ years: a Belgian epidemiological survey using the ICIQ-MLUTS questionnaire. Int J Clin Pract 2015;69:358-365.

21. Société Internationale d'Urologie (SIU), Lower Urinary Tract Symptoms (LUTS) : An International Consultation on Male LUTS. , C. Chapple \& P. Abrams, Editors. 2013. Available at: https://www.siuurology.org/themes/web/assets/files/ICUD/pdf/Male\%20Lower\%20 Urinary\%20Tract\%20Symptoms\%20(LUTS).pdf

22. Malmsten UG, Molander U, Peeker R, et al. Urinary incontinence, overactive bladder, and other lower urinary tract symptoms: a longitudinal population-based survey in men aged 45-103 years. Eur Urol 2010;58:149-156.

23. Roehrborn CG, Siami P, Barkin J, The effects of dutasteride, tamsulosin and combination therapy on lower urinary tract symptoms in men with benign prostatic hyperplasia and prostatic enlargement: 2-year results from the CombAT study. J Urol 2008;179:616-621.

24. Wasson JH, Reda DJ. Bruskewitz RC, et al. A comparison of transurethral surgery with watchful waiting for moderate symptoms of benign prostatic hyperplasia. The Veterans Affairs Cooperative Study Group on Transurethral Resection of the Prostate. N Engl J Med 1995;332:75-79.

25. Zhang T, Wu H, Liu S, et al. Clinical evaluation of tamsulosin in the relief of lower urinary tract symptoms in advanced prostate cancer patients. Int Urol Nephrol 2017;49:1111-1117.

26. McConnell JD, Bruskewitz R, Walsh P, et al. The effect of finasteride on the risk of acute urinary retention and the need for surgical treatment among men with benign prostatic hyperplasia. Finasteride Long-Term Efficacy and Safety Study Group. N Engl J Med 1998;26;338:557-563.

27. Mustafa M, Davis JW, Gorgel SN, Pisters L. Robotic or Open Radical Prostatectomy in Men with Previous Transurethral Resection of Prostate. Urol J 2017; 18;14:2955-2960.

28. Koo KC, Park SU, Rha KH, et al. Transurethral resection of the prostate for patients with Gleason score 6 prostate cancer and symptomatic prostatic enlargement: a risk-adaptive strategy for the era of active surveillance. Jpn J Clin Oncol 2015;45:785-90.

29. Margel D, Nandy I, Wilson TH, et al. Predictors of pathological progression among men with localized prostate cancer undergoing active surveillance: a sub-analysis of the REDEEM study. J Urol 2013;190:2039-2045.

30. Al Edwan G, Fleshner N. 5-alpha reductase inhibitors in patients on active surveillance: do the benefits outweigh the risk? Curr Urol Rep 2013;14:223-226.

31. Giganti F, Moore CM, Robertson NL, et al. MRI findings in men on active surveillance for prostate cancer: does dutasteride make MRI visible lesions less conspicuous? Results from a placebo-controlled, randomised clinical trial. Eur Radiol 2017;27:4767-4774. 